\title{
THE ADDITIVITY OF THE LEBESGUE AREA
}

\author{
J. W. T. YOUNGS
}

A triple of continuous functions $T: x^{i}\left(u^{1}, u^{2}\right), i=1,2,3$, defined on a closed square $Q\left[0 \leqq u^{1} \leqq 1,0 \leqq u^{2} \leqq 1\right]$ represents a surface $\mathfrak{S}$ $(1.6,1.17,1.21){ }^{1}$ If $r$ is any closed rectangle in $Q$ then we may speak of the triple $T_{r}$ consisting of the above triple $T$ with its range of definition restricted to $r$. This triple generates a surface $\mathbb{S}(r)$. If $r_{1}$ and $r_{2}$ have no interior points in common, and $r_{1}+r_{2}=Q$, it is natural to hope that $L(\widetilde{S})=L\left(\widetilde{S}\left(r_{1}\right)\right)+L\left(\widetilde{S}\left(r_{2}\right)\right)$ where $L$ is the symbol used to indicate the Lebesgue area (3.13). This statement is certainly true whenever the Lebesgue area is given by the standard integral formula, since the Lebesgue integral is additive. However, in general it is false. It may be said that this note is concerned with the statement that if the triple is constant on $r_{1} \cdot r_{2}$, the Lebesgue area is additive.

Stated in this fashion the theorem may appear to be new. Actually it is but a very special case of a fundamental problem in the theory of area. The classical conjecture is that the area is additive under the much weaker requirement that the triple be rectifiable on $r_{1} \cdot r_{2}$. No proof of this conjecture has, to our knowledge, appeared. (In this connection see McShane [1, p. 138].)

In relation to existing literature the theorem of this note is included in a more general theorem due to Morrey [1]. In fact, the first case in Morrey's proof [1, p. 314] is essentially the theorem of this paper. A discrepancy in his argument was rectified by Radó and Reichelderfer [1] in an independently interesting discussion of stretching processes.

This result, therefore, is not new.

The point of this treatment is the utter simplicity of the stretching process here employed. The development serves to make a known result more accessible.

THEOREM 1. If a polyhedron $\mathfrak{P}(3.11,1.21)$ has a quasi-linear (1.7) representation $T: x(u), u \in Q$, such that the image of the side $s\left[u^{1}=1\right.$, $\left.0 \leqq u^{2} \leqq 1\right]$ is interior to a sphere of diameter less than $\epsilon$ about the point $a\left[a^{1}, a^{2}, a^{3}\right]$, then there exists a polyhedron $\mathfrak{B}^{*}$ with quasi-linear representation $T^{*}: x^{*}(u), u \in Q$, such that

Presented to the Society, February 27, 1943; received by the editors March 23, 1943.

1 The notation and terminology of this note are largely due to Rado [1]; in fact, numbers in parentheses refer the reader to appropriate paragraphs in his paper. Numbers in brackets refer to the bibliography. 
(1) $E\left(\mathfrak{B}^{*}\right)=E(\mathfrak{B}) .^{2}$

(2) $x^{*}(u)=a$ for $u \in s$.

(3) $\left\|x^{*}(u)-x(u)\right\|<\epsilon$, hence $d\left(\mathfrak{B}^{*}, \mathfrak{P}\right)<\epsilon(1.1,1.22)$.

Proof. Define $x_{\alpha}\left(u^{1}, u^{2}\right)=x\left(\alpha u^{1}, u^{2}\right), \alpha>1$. Since $T$ is continuous we may choose $\alpha$ so that $\left\|x_{\alpha}(u)-x(u)\right\|<\epsilon$, for $u \in R_{\alpha}\left[0 \leqq u^{1} \leqq 1 / \alpha\right.$, $\left.0 \leqq u^{2} \leqq 1\right]$, and further the image of $S=Q-R_{\alpha}$ under $T$ is in a sphere of diameter less than $\epsilon$ about $a$. The representation $x_{\alpha}(u)$ is certainly quasi-linear in $R_{\alpha}$.

Define $x^{*}(u)$ to be equal to $x_{\alpha}(u)$ on $R_{\alpha}$. Now $x^{*}(u)$ is a quasi-linear representation of $\mathfrak{P}$ on $R_{\alpha}$.

We have yet to define $x^{*}(u)$ on the rest of $Q$. There is a set of intervals $I_{1}, \cdots, I_{n-2}$ on $\left[u^{1}=1 / \alpha, 0 \leqq u^{2} \leqq 1\right]$ such that $x^{*}(u)$ is linear on each of them and consecutive $I$ 's abut. Their end points, in order, are

$$
p_{0}[1 / \alpha, 0], p_{1}, \cdots, p_{n-2}[1 / \alpha, 1] .
$$

Add to this sequence $p_{n-1}[1,1]$ and $p_{n}[1,0]$.

Let $p$ be the point $[(\alpha+1) / 2 \alpha,-1]$ and consider the segments $\left(p, p_{k}\right), k=1, \cdots, n-1$. Each segment cuts $\left[1 / \alpha \leqq u^{1} \leqq 1, u^{2}=0\right]$ in exactly one point $q_{k}$. Join $p_{k}$ to $q_{k+1}, k=1, \cdots, n-2$. The rectangle $S$ is now subdivided into $2 n-2$ triangles whose vertices are in the set $p_{0}, \cdots, p_{n}, q_{1}, \cdots, q_{n-1}$.

The triple $x^{*}(u)$ has been defined at the vertices $p_{0}, \cdots, p_{n-2}$.

Let $x^{*}\left(p_{n-1}\right)=x^{*}\left(p_{n}\right)=a$, and $x^{*}\left(q_{k}\right)=x^{*}\left(p_{k}\right), k=1, \cdots, n-1$.

Since the mapping is defined at the vertices of each triangle of the subdivision there is a unique linear extension over each triangle. The extensions will match along the side two triangles may have in common, and hence we have a quasi-linear ${ }^{3}$ extension on $S$. Define $x^{*}(u)$ over $S$ to be this extension.

Now $T^{*}: x^{*}(u)$ is quasi-linear on $Q$ and so represents a polyhedron $\mathfrak{B}^{*}$.

It is important to notice that the map of each of the triangles in $S$ is a segment or a point and hence contributes zero area. Therefore $E\left(\mathfrak{P}^{*}\right)=E(\mathfrak{P})$.

Secondly, $x^{*}(u)=x^{*}\left(p_{n}\right)=x^{*}\left(p_{n-1}\right)=a$ for $u \in s$.

Finally, $T^{*}(S) \subset T(S)+\left[\right.$ the segment from $T\left(p_{n-1}\right)$ to $\left.a\right]$. But $T(S)$

${ }^{2} E(\mathfrak{B})$ is the elementary area of the polyhedron $\mathfrak{B}$, that is, the area in the ordinary geometric sense. It is not necessary, for the purpose of this note, to know that this coincides with the Lebesgue area. The fact that this is so, though sometimes ignored, is by no means obvious (3.20).

${ }^{3}$ It is important to notice that a quasi-linear representation need not be a map which carries each triangle of the subdivision into a nondegenerate triangle. The point set covered by a quasi-linear map may, for example, be a single point. 
is contained in the sphere of diameter $\epsilon$ about $a$. Therefore $T^{*}(S)$ is in the same sphere. Now in view of our choice of $\alpha$ it follows that $\left\|x^{*}(u)-x(u)\right\|<\epsilon$ for $u \in Q$.

REMARK. The theorem may be stated with any rectangle $R$ replacing the square $Q$.

Let us turn at once to see how this stretching process is applied in the proof of the additivity result.

Consider a continuous triple $T: x(u), u \in Q$. Let $Q$ be divided by a line $u^{1}=c$ into two rectangles $R_{1}\left[0 \leqq u^{1} \leqq c, 0 \leqq u^{2} \leqq 1\right]$ and $R_{2}\left[c \leqq u^{1} \leqq 1,0 \leqq u^{2} \leqq 1\right]$. Let $T_{i}$ be the triple $x(u), u \in R_{i}, i=1,2$. Now $T$ is a representation of a surface $\subseteq$, while $T_{i}$ is a representation of a surface $\widetilde{S}_{i}, i=1,2$. It is both well known and easy to see that $L\left(\Im_{)} \geqq L\left(\Im_{1}\right)+L\left(\Im_{2}\right)\right.$. The reverse inequality is usually false. We are interested in a case in which it is true, making the Lebesgue area additive.

THEOREM 2. If $x(u)$ is constant on $u^{1}=c$, then $L\left(S_{)}\right)=L\left(\Im_{1}\right)+L\left(\Im_{2}\right)$.

Proof. Grant, for the moment, the existence of two sequences of polyhedra $\left\{{ }_{1} \mathfrak{P}_{n}\right\}$ and $\left\{{ }_{2} \mathfrak{B}_{n}\right\}$, having the property that for $i=1,2$, $E\left({ }_{i} \mathfrak{B}_{n}\right) \rightarrow L\left(\mathfrak{S}_{i}\right)$, and each ${ }_{i} \mathfrak{\Re}_{n}$ has a quasi-linear representation ${ }_{i} x_{n}(u)$ on $R_{i}$ such that $\left\|_{i} x_{n}(u)-x(u)\right\|<1 / n$ for $u \in R_{i}$.

The justification of this will be taken up presently.

It is enough to obtain a sequence of polyhedra $\left\{\mathfrak{B}_{n}\right\}$ with quasilinear representation $x_{n}(u)$ on $Q$ such that $E\left(\mathfrak{B}_{n}\right)$ is within $1 / n$ of $E\left({ }_{1} \mathfrak{B}_{n}\right)+E\left({ }_{2} \mathfrak{B}_{n}\right)$, and $\mathfrak{P}_{n} \rightarrow \subseteq$.

As a matter of fact, we are going to obtain the stronger result, $E\left(\mathfrak{P}_{n}\right)=E\left({ }_{1} \mathfrak{P}_{n}\right)+E\left({ }_{2} \mathfrak{P}_{n}\right)$, and $\mathfrak{B}_{n} \rightarrow \mathfrak{S}$.

The theorem will then follow, since by the definition of the Lebesgue area, $L(\subseteq) \leqq \lim \inf E\left(\mathfrak{P}_{n}\right)=\lim \inf \left[E\left({ }_{1} \mathfrak{P}_{n}\right)+E\left({ }_{2} \mathfrak{P}_{n}\right)\right]=\lim E\left({ }_{1} \mathfrak{P}_{n}\right)$ $+\lim E\left({ }_{2} \mathfrak{R}_{n}\right)=L\left(\Im_{1}\right)+L\left(\Im_{2}\right)$.

The process by which the sequence of polyhedra $\left\{\mathfrak{P}_{n}\right\}$ is obtained is based on Theorem 1. Were we to define $x_{n}(u)$ to be ${ }_{i} x_{n}(u)$ on $R_{i}$ the $x_{n}(u)$ would fail to represent the required sequence of polyhedra only by virtue of the fact that continuity, in general, would be lacking along $u^{1}=c$. Theorem 1 essentially enables us to alter ${ }_{1} x_{n}(u)$ and ${ }_{2} x_{n}(u)$ slightly as to position and not at all as to area so that they will match along $u^{1}=c$.

Consider any ${ }_{i} \mathfrak{P}_{n}$ and its quasi-linear representation ${ }_{i} x_{n}(u), u \in R_{i}$. Theorem 1 guarantees the existence of a polyhedron ${ }_{i} \mathfrak{B}_{n}{ }^{*}$ with quasilinear representation $x_{n}{ }^{*}(u), u \in R_{i}$, such that:

(1) $E\left({ }_{i} \mathfrak{P}_{n}^{*}\right)=E\left({ }_{i} \mathfrak{P}_{n}\right)$.

(2) ${ }_{i} x_{n}^{*}(u)=x(u)$, a constant, on $\left[u^{1}=c, 0 \leqq u^{2} \leqq 1\right]$. 
(3) $\left\|x_{i} x_{n}(u)-{ }_{i} x_{n}(u)\right\|<2 / n$.

Now define $T_{n}: x_{n}(u)={ }_{i} x_{n}^{*}(u), u \in R_{i}, i=1,2$.

This is a continuous triple due to condition (2), and so it is a quasilinear triple representing a polyhedron $\mathfrak{B}_{n}$. We shall show that $\left\{\mathfrak{P}_{n}\right\}$ has all the desired properties.

First, $E\left(\mathfrak{B}_{n}\right)=E\left({ }_{1} \mathfrak{P}_{n}\right)+E\left({ }_{2} \mathfrak{B}_{n}\right)$ since the elementary area is additive.

Next, for $i=1,2$ :

$$
\begin{aligned}
\left\|x_{n}(u)-x(u)\right\| & =\left\|_{i} x_{n}^{*}(u)-x(u)\right\| \text { on } R_{i} \\
& \leqq\left\|_{i} x_{n}^{*}(u)-{ }_{i} x_{n}(u)\right\|+\left\|_{i} x_{n}(u)-x(u)\right\| \text { on } R_{i} \\
& \leqq 2 / n+1 / n \text { on } Q .
\end{aligned}
$$

Therefore $x_{n}(u)$ converges uniformly to $x(u)$ on $Q$ and hence $\left\{\mathfrak{P}_{n}\right\}$ certainly converges to $\widetilde{\varsigma}$.

It now remains but to consider the statement deferred in the first paragraph of the proof.

Briefly the problem amounts to this: Given a representation $x(u)$ of a surface $\mathbb{S}$ on a rectangle $R$ we need to know that there exists a polyhedron $\mathfrak{B}$ whose area is close to the area of $\mathfrak{S}$ and admits of a quasilinear representation on $R$ which is close to the given representation of $\Im$.

Given $\epsilon>0$, by the definition of the Lebesgue area (3.13) there is a polygon $\mathfrak{P}$ such that $d(\mathfrak{B}, \mathfrak{S})<\epsilon$ and $|E(\mathfrak{B})-L(\mathfrak{S})|<\epsilon$.

Since $\mathfrak{P}$ is a polyhedron it has a quasi-linear representation $\bar{x}(u)$, $u \in B$. The set $B$ is the closure of a Jordan region with polygonal boundary. The set $B$ may be $R$ but this is by no means certain, or even useful. By the definition of distance (1.17) there is a topological transformation $\tau(R)=B$ such that $\|x(u)-\bar{x}(\tau(u))\|<\epsilon$.

The continuity of $\bar{x}(u)$ guarantees that there is a $\delta>0$ such that all maps $\mu(R)=B$ satisfying $\|\mu(u)-\tau(u)\|<\delta$ have the property that $\|\bar{x}(\mu(u))-\bar{x}(\tau(u))\|<\epsilon$. Hence $\|x(u)-\bar{x}(\mu(u))\|<2 \epsilon$.

We can select $\mu(u)$ so that in addition to satisfying the above inequality it is quasi-linear and topological on $R$. The proof of this assertion follows as a result of some remarks of Franklin and Wiener [1, pp. 764-766].

Lemma (FrankLIN AND WIENER). Given $\epsilon>0$ and $a$ topological transformation $\tau(s)=S$ (where $s$ and $S$ are closed squares) we may subdivide $s$ and $S$ into corresponding convex polygons . . having the property that any topological transformation $\mu(s)=S$ which maps each polyhedron of $s$ onto the corresponding polyhedron of $S$ is at a distance less than $\epsilon$ from $\tau$. 
The reader will readily make the necessary adjustments from $s$ to $R$ and $S$ to $B$. The convex polygons mentioned above may be chosen as triangles and a $\mu$ may be exhibited which is linear on the triangles of $R$.

Now $\bar{x}(\mu(u))$ is clearly quasi-linear on $R$. Moreover, it is a representation of $\mathfrak{P}$, and so $\mathfrak{B}$ has a quasi-linear representation on $R$ within $2 \epsilon$ of the given representation of $\subseteq$. Since $|E(\mathfrak{P})-L(\widetilde{S})|<\epsilon$ the proof is complete.

We have incidentally proved the independently important theorem:

Theorem 3. Any polyhedron has a quasi-linear representation on a rectangle.

REMARK. The square $Q$ in the additivity theorem can be replaced by a simply connected Jordan region and its boundary, the dividing line can then be replaced by any crosscut on which the triple is constant.

It is possibly of some interest, in conclusion, to compare the surface stretching done here in Theorem 1 with that of the paper by Radó and Reichelderfer. Briefly, their process stretches a surface directly towards a fixed point. Each point of the surface sufficiently close to the fixed point is moved along the ray to the fixed point.The stretching is managed, however, so that the increase in area, though generally positive, is ingeniously kept within bounds. Here the stretching is by no means directly towards the fixed point, and it is precisely the rather circuitous route taken to the point which enables us to keep the added area equal to zero. If a polyhedron undergoes their stretching process it will, in general, no longer be a polyhedron; the stretching done here will preserve the polyhedral character of the surface. On the other hand their stretching process applies to situations which this cannot touch. For example, it will deal with the case in which $r_{1}$ is a square interior to $Q$, the set $r_{2}=\overline{Q-r_{1}}$, and the triple is constant on $r_{1} \cdot r_{2}$.

\section{BIBLIOGRAPHY}

P. FrankLin AND N. Wiener

1. Analytic approximations to topological transformations, Trans. Amer. Math. Soc. vol. 28 (1926) pp. 762-785.

E. J. McShane

1. On a certain inequality of Steiner, Ann. of Math. vol. 33 (1932) pp. 125-138.

C. B. MORREY

1. An analytic characterization of surfaces of finite Lebesgue area. II, Amer. J. Math. vol. 58 (1936) pp. 312-322. 
TiBor Radó

1. On the semi-continuity of double integrals in parametric form, Trans. Amer. Math. Soc. vol. 51 (1942) pp. 336-361.

TiBor Radó AND P. REICHELdERFER

1. On a stretching process for surfaces, Amer. J. Math. vol. 61 (1939) pp. 645-650.

PuRdue University

\section{SOLUTION OF THE "PROBLÈME DES MENAGES”}

\section{IRVING KAPLANSKY}

The problème des ménages asks for the number of ways of seating $n$ husbands and $n$ wives at a circular table, men alternating with women, so that no husband sits next to his wife. Despite the considerable literature devoted to this problem (cf. the appended bibliography), the following simple solution seems to have been missed.

It is convenient first to solve two preliminary problems, perhaps of some interest in themselves.

LEMma 1. The number of ways of selecting $k$ objects, no two consecutive, from $n$ objects arrayed in a row is ${ }_{n-k+1} C_{k}$.

Let $f(n, k)$ be the desired number. We split the selections into two subsets: those which include the last of the $n$ objects and those which do not. The former are $f(n-2, k-1)$ in number (since further selection of the second last object is forbidden); the latter are $f(n-1, k)$ in number. Hence

$$
f(n, k)=f(n-1, k)+f(n-2, k-1),
$$

and, combining this with $f(n, 1)=n$, we readily prove by induction that $f(n, k)={ }_{n-k+1} C_{k}$.

Lemma 2. The number of ways of selecting $k$ objects, no two consecutive, from $n$ objects arrayed in a circle is ${ }_{n-k} C_{k} n /(n-k)$.

This differs from the preceding problem only in the imposition of the further restriction that no selection is to include both the first and last objects; and the number of such selections which are otherwise acceptable is $f(n-4, k-2)$. Hence the desired result is $f(n, k)$ $-f(n-4, k-2)={ }_{n-k} C_{k} n /(n-k)$.

Presented to the Society, September 13, 1943; received by the editors May 4, 1943. 